\title{
Some Remarks on Real and Algebraic Cobordism
}

\author{
PO HU ${ }^{1}$ and IGOR KRIZ \\ ${ }^{1}$ Department of Mathematics, University of Chicago, 5734 S. University Avenue, Chicago, IL \\ 60637, U.S.A. and Department of Mathematics, University of Michigan, 2074 East Hall, 525 East \\ University Avenue, Ann Arbor, MI 48109-1109, U.S.A.
}

(Received: September 2000)

Abstract. We investigate connections between Real cobordism, algebraic cobordism, quadratic forms, the Rost Motive, Morava $K(n)$-theories and analogues of homotopy classes of Hopf invariant 1 .

Mathematics Subject Classifications (2000): 55N22, 19L41, 10C05, 11E81, 14F42, 19D99

Key words: Real cobordism, algebraic cobordism, quadratic forms, Rost motive, Morava $K$-theories

\section{Introduction}

In this paper, we will explore some analogies between Real cobordism investigated by the authors [13] (and others previously, see Landweber [17], Araki [2]), and algebraic cobordism introduced by Voevodsky in his proof of the Milnor conjecture. We will use these analogies to derive new results and conjectures in both worlds. In particular, we will exhibit an analogue of the Rost Motive [25] for free Real Milnor manifolds, and, on the other hand, formulate an analogue of the Hopf invariant 1 problem in the category of spaces over a field $k$.

Real cobordism is defined by considering the action of complex conjugation on complex cobordism. More precisely, $\mathbb{Z} / 2$ acts by conjugation on $B U(n)$ and the Thom space $B U(n)^{\gamma_{n}}$ of the universal complex $n$-bundle, and we have a map

$$
\Sigma^{\mathbb{C}} B U(n)^{\gamma_{n}} \rightarrow B U(n+1)^{\gamma_{n+1}} .
$$

Here, of course, $\mathbb{Z} / 2$-equivariantly, $\mathbb{C}=1+\alpha$ where 1 is the trivial one-dimensional real representation of $\mathbb{Z} / 2$, and $\alpha$ is the sign representation. Thus, (1.1) defines a spectrum $M \mathbb{R}$ indexed over the complete $\mathbb{Z} / 2$-universe, or, in other words, a generalized cohomology theory $M \mathbb{R}^{k+\ell \alpha} X, k, \ell \in \mathbb{Z}$ on $\mathbb{Z} / 2$-equivariant spaces $X$. The double indexing comes from the fact that the theory is stable under suspension both by the trivial and the sign representations of $\mathbb{Z} / 2$ (see [18]).

Voevodsky's algebraic cobordism is a similar construction in the category of spaces over a field $k$ (called $\mathbb{A}^{1}$-homotopy theory, see [22], [28]). If $B G L(n)^{\gamma_{n}}$ is, again, the Thom space of the canonical $n$-bundle, one obtains an analogue of (1.1)

$$
\Sigma^{\mathbb{A}^{1}} B G L(n)^{\gamma_{n}} \rightarrow B G L(n+1)^{\gamma_{n+1}} .
$$


Now Voevodsky also has a decomposition of $S^{\mathbb{A}^{1}}$

$$
S^{\mathbb{A}^{1}} \simeq S_{s}^{1} \wedge S_{t}^{1} .
$$

Here $S_{s}^{1}$ is $\mathbb{A}^{1} /\{0,1\}$ and $S_{t}^{1}$ is $\mathbb{G}_{m}$ with base point 1 . Thus, (1.2) gives rise to a spectrum $M G L$ over $k$ ( $\mathbb{P}^{1}$-spectrum), i.e. a generalized cohomology theory in the category of spaces over $k$. Further, (1.3) shows that such theories $E$ should also be indexed by two numbers, called the dimension and the twist. The common notation is

$$
E^{u, v}(X)=\left[X,\left(\left(S_{s}^{1}\right)^{\wedge(u-v)} \wedge\left(S_{t}^{1}\right)^{v} \wedge E\right)_{0}\right]
$$

where the right-hand side indicates homotopy classes of maps of $k$-spaces, and the subscript indicates the infinite loop space (over $k$ ) associated with a $\mathbb{P}^{1}$-spectrum.

There is a clear analogy between the $\mathbb{Z} / 2$-equivariant and algebraic situations. This analogy, is, of course, not coincidental: in the case when $k \subseteq \mathbb{R}$, we have a realization functor $t_{\mathbb{R}}$ (see [22]) from $k$-spaces to $\mathbb{Z} / 2$-equivariant spaces: for a variety $X, t_{\mathbb{R}}(X)$ is the space $X_{\mathbb{C}}$ with Galois action of $\mathbb{Z} / 2 \cong \operatorname{Gal}(\mathbb{C} / \mathbb{R})$. We have

$$
t_{\mathbb{R}} S_{s}^{1} \simeq S^{1}, t_{\mathbb{R}} S^{1_{t}} \simeq S^{\alpha},
$$

and consequently $t_{\mathbb{R}}$ also extends to spectra, thus forming a functor from $k$-spectra to $\mathbb{Z} / 2$-equivariant spectra.

Note, however, that the algebraic situation is more general. (1.2), (1.3), (1.4) make sense even if $k$ is an arbitrary field, not necessarily contained in $\mathbb{R}$. It is this generality which seems to afford the most intriguing unexplored directions.

Since the present paper explores analogies between the Real and algebraic situations, this clearly calls for a unified notation. After debating this, we decided to adopt the topological notation and change the algebraic one here, since the topological notation is closer to our thinking. Thus, also for Voevodsky's $k$-spaces, we shall write in (1.3)

$$
\begin{aligned}
& S^{1}=S_{s}^{1} \simeq \mathbb{A}^{1} /\{0,1\}, \\
& S^{\alpha}=S_{t}^{1} \simeq \mathbb{G}_{m},
\end{aligned}
$$

and

$$
E^{k+\ell \alpha}=\left[X,\left(\left(S^{1}\right)^{\wedge k} \wedge\left(S^{\alpha}\right)^{\wedge \ell} \wedge E\right)_{0}\right],
$$

which is

$$
E^{k+\ell, \ell} X
$$

in the traditional notation. For example, if $H$ denotes ordinary motivic cohomology, then

$$
H^{1+\alpha} X=H^{2,1} X=H^{2}(X, \mathbb{Z}(1))=H^{1}\left(X, \mathbb{G}_{m}\right)=\operatorname{Pic}(X) .
$$


In Section 2 of this paper, we briefly develop the theory of algebraically oriented spectra in parallel with the existing theories of complex- and Real- oriented spectra (see [1], [24], [3] and [13]). This is an obvious idea (hardly original work), but it is essential to what follows in the paper, and it is not too long.

In particular, we show that maps of ring spectra $M G L \rightarrow E$ are in bijective correspondence with algebraic orientations on $E$, and construct $B P G L$. Further, $E_{\infty}$ structure of $M G L$ can be used to construct the usual menagerie of spectra derived from $M G L$, in particular Morava $K(n)$-theories. In particular, it is fun to note that the Real and algebraic analogues of $K$-theory is Atiyah's Real $K R$-theory [5] and algebraic $K$-theory, and the Real and algebraic analogues of singular cohomology theory $H$ is $\mathbb{Z} / 2$-equivariant cohomology with constant Mackey coefficients, and Motivic cohomology.

In Section 3, we formulate some basic conjectures about $M G L$ : a transversality hypothesis analogous to the Real case, which would say that $M G L$-cohomology groups of smooth algebraic varieties would vanish for $k>\ell$, and also describe the groups for $k=\ell$. We also formulate a structure conjecture for $B P G L$ : this conjecture is analogous to what is known to be true in the Real and complex cases: there should be an 'Adams tower' building $B P G L_{\ell}^{\wedge}$ from copies of $H \mathbb{Z} / \ell_{\text {Mot }}$ indexed by Milnor words. An analogue of the structure conjecture for Morava $K(n)$-theories was formulated by Voevodsky [27], and used in his original approach to the Milnor conjecture.

The structure conjecture might be a good substitute for a Lichtenbaum-Quillentype completion conjecture, which seems harder to formulate for $B P G L$. (Both of these statements give essentially the same information in the Real case.)

In Section 4, we return to the Real case. We prove an analogue of the theorem of Markus Rost [25], splitting a certain motive (the Rost motive) off the cohomology of a Pfister quadric. This is the main calculational ingredient in Voevodsky's proof of the Milnor conjecture [28]. In the Real case, the conclusion is, of course, weaker (we are giving up the algebraic structure), but the hypothesis is much weaker also. The result holds for every free Real Milnor manifold. Moreover, considering the analogue of the Rost motive in the Real case reveals a surprizing connection with other concepts, including the coefficients of Real Morava $K(n)$-theory $K \mathbb{R}(n)$, and the Hopf invariant 1 theorem.

Concretely, the $\mathbb{Z} / 2$-equivariant Rost motive is the Mackey cohomology of $S\left(\left(2^{n+1}-1\right) \alpha\right)$. One finds that the $K(n)$-theoretical analogue of the $\mathbb{Z} / 2$-equivariant Rost motive is

$$
K \mathbb{R}(n) \wedge S\left(\left(2^{n+1}-1\right) \alpha\right)=K \mathbb{R}(n) \vee \Sigma^{2^{n}(1-\alpha)} K \mathbb{R}(n) .
$$

Also, one can consider a cofibration

$$
\Sigma^{\left(2^{n}-1\right)(1+\alpha)}\left(S^{0}\right)^{\perp} \rightarrow S\left(\left(2^{n+1}-1\right) \alpha\right)_{+} \rightarrow S^{0},
$$

in which case the Hopf invariant 1 problem can be reformulated as

$$
S\left(2^{n} \alpha\right)_{+} \wedge\left(S^{0}\right)^{\perp} \simeq S\left(2^{n} \alpha\right)_{+} .
$$


The balance of the paper is dedicated to developing and exploring analogues of (1.6), (1.7) and (1.8) in the algebraic universe of $k$-spaces. In Section 5, we find an analogue of $S\left(\left(2^{n+1}-1\right) \alpha\right)_{+}$, i.e. a $k$-spectrum whose homology is the Rost motive. We also define 'hypersymplectic Morava $K(n)$-theory'

$$
K(n)^{\perp}=K(n) \wedge\left(S^{0}\right)^{\perp} .
$$

This is motivated by a striking example of Quillen [23] for $n=1$, when the Rost spectrum is just a projective conic, and $K^{\perp}$ is symplectic $K$-theory, i.e. the algebraic $K$-theory of the central simple $k$-algebra associated with the conic. One can see here why in the Real case all we are seeing is a shift (1.6): Real symplectic $K$-theory is, by Bott periodicity, just a shift of Real $K$-theory.

In Section 6, we look for an algebraic analogue of the periodicity properties of coefficients of Real cobordism $M \mathbb{R}$. This leads to another conjecture about the coefficients of $M G L$, which we call the periodicity conjecture. Assuming this conjecture and the transversality hypothesis, we prove an analogue of Rost's splitting of the motives of Pfister quadrics with motivic cohomology replaced by algebraic Morava $K(n)$-theory. We also show how these considerations motivate questions about the Picard group of the $\mathbb{A}^{1}$-stable homotopy category, which was further studied by the first author [12].

In Section 7, we define and investigate the analogue of $S\left(2^{n} \alpha\right)_{+}$and the algebraic analogue of the Hopf invariant 1 problem. We also introduce the concept of a non-associative division algebra with a given quadratic form $Q$ as norm, and show how the existence of such an algebra implies a positive answer to the Hopf invariant 1 question. We also show that for $k \subseteq \mathbb{R}$, the algebraic Hopf invariant 1 problem reduces to the classical (topological) Hopf invariant 1 theorem. But, for a field where -1 is a sum of squares, the question remains open.

Throughout this paper, we focus on the prime $\ell=2$, since the Hopf invariant problem as well as the Rost motive are special to that prime. Still, perhaps, the most striking question is what, if any, analogue this theory has for the primes $\ell>2$.

\section{Algebraically Oriented Spectra}

In this section, we shall consider commutative associative ring spectra $E$ in the category of spaces over $k$ [22], [28]. Assume that the field $k$ is of characteristic 0 throughout this paper, unless explicitly stated. Recall that, for a generalized cohomology theory $E$, a bundle $\gamma$ on a space $X$ is $E$-orientable if there exists a class

$$
u \in E^{\star} X^{\gamma},
$$

called the Thom class, where the superscript $\gamma$ indicates Thom space, such that for every map of spaces

$$
Y \stackrel{f}{\longrightarrow} X,
$$


the map

$$
\tilde{E}^{\star}\left(X^{f^{*} \gamma}\right) \stackrel{\theta^{*}}{\longleftarrow} E^{\star}(Y) \otimes E^{\star}\left(Y^{f^{*} \gamma}\right) \stackrel{1 \otimes f^{*} u}{\longleftarrow} E^{\star}(Y)
$$

is an isomorphism where the map $\theta^{*}$ is induced by the Thom diagonal $Y^{f^{*} \gamma} \rightarrow$ $Y^{f^{*} \gamma} \wedge Y_{+}$. This definition works in the non-equivariant, equivariant and algebraic contexts.

DEFINITION 2.1. We say that $E$ is algebraically orientable if for every $n$, the universal $n$-bundle $\gamma^{n}$ on $B G L_{n}$ is $E$-orientable. If $E$ is algebraically orientable, by an algebraic orientation we shall mean a class $u \in E^{1+\alpha} \mathbb{P}^{\infty}$ which restricts to $1 \in E^{1+\alpha} \mathbb{P}^{1}$ via the inclusion $\mathbb{P}^{1} \subset \mathbb{P}^{\infty}$. Such class is automatically a Thom class of $\gamma^{1}$.

COMMENT. Bryan Johnston, following the method of [7], recently found an argument showing that, analogously to the Real case, algebraic orientability can be deduced from the existence of an algebraic orientation in the sense of the above Definition.

PROPOSITION 2.2. Let $E$ be an algebraically oriented spectrum over $k$ with orientation $u$. Then

$$
E^{\star} \mathbb{P}^{\infty}=E^{\star}[[u]] .
$$

More generally,

$$
E^{\star}(\underbrace{\mathbb{P}^{\infty} \times \cdots \times \mathbb{P}^{\infty}}_{n \text { times }})=E^{\star}\left[\left[u_{1}, \ldots, u_{n}\right]\right] .
$$

Proof. Since we have a cofibration $\mathbb{P}^{n-1} \rightarrow \mathbb{P}^{n} \rightarrow S^{n(1+\alpha)}$, we obtain, using the associated $E^{\star}$-long exact sequence, and the orientation class $u$, inductively $E^{\star} \mathbb{P}^{n}=$ $E^{\star}[u] / u^{n+1}$. Passing to limit and realizing that $\lim ^{1}=0$, we obtain (2.3). (2.4) is proved analogously.

We see that

$$
E^{\star} \lim _{\rightarrow}\left(\mathbb{P}^{\infty}\right)^{n}=\lim _{\leftarrow} E^{\star}\left(\mathbb{P}^{\infty}\right)^{n}=E^{\star}\left[\left[u_{1}, u_{2}, u_{3}, \ldots\right]\right],
$$

where the morphisms in the direct limit on the left-hand side of (2.5) are induced by inclusions of one point $* \rightarrow \mathbb{P}^{\infty}$. (It does not matter which point, since any two points are on an affine line.)

PROPOSITION 2.6. We have

$$
E^{\star} B G L_{n}=E^{\star}\left[\left[c_{1}, \ldots, c_{n}\right]\right],
$$




$$
E^{\star} B G L=E^{\star}\left[\left[c_{1}, \ldots, c_{n}, \ldots\right]\right],
$$

where in the inclusions $B G L_{n} \subseteq\left(\mathbb{P}^{\infty}\right)^{n}, B G L \subseteq \lim _{\rightarrow}\left(\mathbb{P}^{\infty}\right)^{n}, c_{i}$ are identified with the elementary symmetric polynomials (resp. series) in the $u_{i}$ 's.

Proof. (2.8) is proved from (2.7) by passage to colimits. (2.7) is an induction on $n$. Consider the Gysin sequence

$$
B G L_{n-1} \rightarrow B G L_{n} \rightarrow\left(B G L_{n}\right)^{\gamma^{n}}
$$

(where the right-hand side denotes the Thom space). This gives an exact sequence

$$
E^{\star} B G L_{n-1} \leftarrow E^{\star} B G L_{n} \leftarrow \Sigma^{n(1+\alpha)} E^{\star} B G L_{n} .
$$

The right-hand arrow is multiplication by the Euler class, which we denote by $c_{n}$. Now applying $E^{\star}$ to the diagram

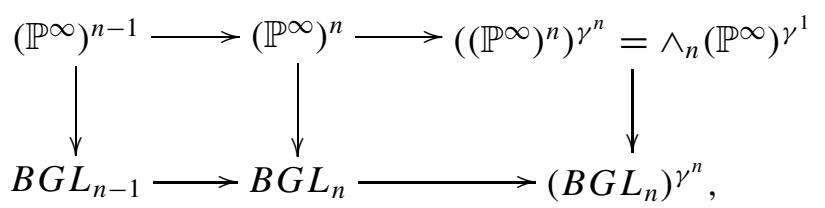

we obtain

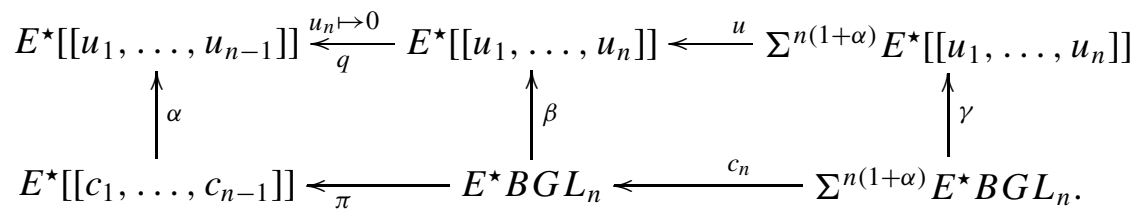

where $u=u_{1} \cdots u_{n}$.

To proceed, we need

CLAIM 2.12. The map $\pi$ is surjective, the map $\beta$ is injective.

Proof. Denote by $B G L_{n, m}$ the subspace of $B G L_{n}$ consisting of all $n$-dimensional affine subspaces of $\mathbb{A}^{\infty}$ which have a nonzero intersection with $\mathbb{A}^{m} \subset$ $\lim _{\rightarrow} \mathbb{A}^{k}=\mathbb{A}^{\infty}$. Then the Gysin sequences restrict to

$$
B G L_{n-1} \rightarrow B G L_{n, m} \rightarrow B G L_{n, m-1}^{\gamma^{n}} .
$$

Note that $B G L_{n, 1} \cong B G L_{n-1}$. Thus, we obtain a diagram analogous to (2.10)

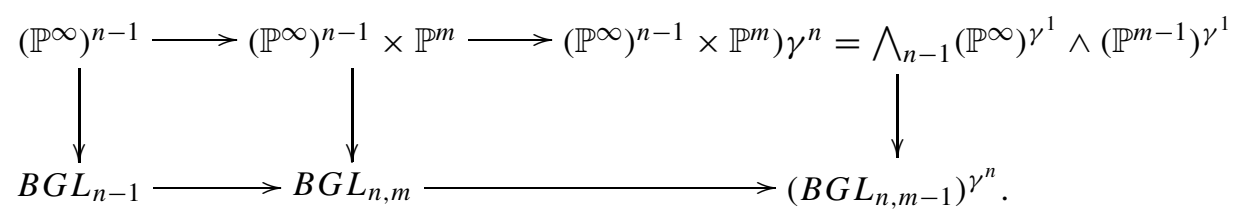


Now applying $E^{\star}$, we get by induction a diagram analogous to (2.11)

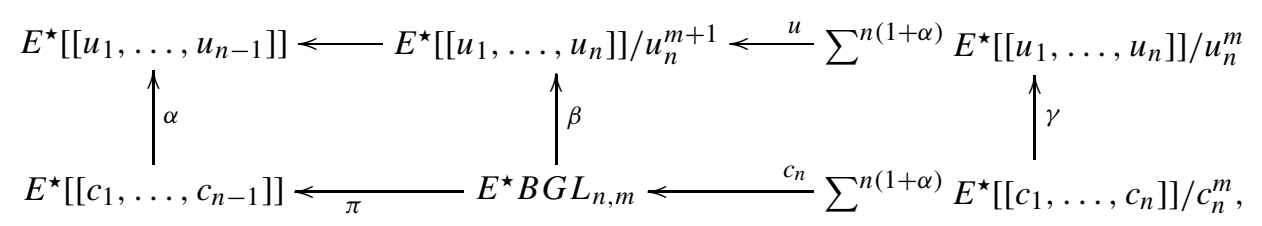

where $u=u_{1} \cdots u_{n}$. We see that $\gamma$ is injective, and so is $\left(u_{1} \cdots \cdots u_{n}\right) \cdot \gamma$. Hence, $c_{n}$ is injective (and hence $\pi$ is surjective and $\beta$ is injective). The Claim follows by a limit argument.

To conclude the proof of the Proposition, note that since the bottom row of (2.11) is a short exact sequence, $E^{\star} B G L_{n}$ is generated by the preimages of $c_{1}, \ldots$, $c_{n} \in E^{\star} B G L_{n-1}$, and $c_{n}$. But the preimages of $c_{i}$ must map by $q \beta=\alpha \pi$ to the $i$ 'th elementary symmetric polynomial. Since, however, $\beta$ is easily seen to also be a symmetric polynomial, it must be the $i$ 'th elementary symmetric polynomial.

PROPOSITION 2.14. (a) Under the description of Proposition (2.6), maps

$$
\Sigma^{\infty} B G L_{+} \rightarrow E
$$

which are maps of ring spectra correspond to series of the form

$$
\prod_{i=1}^{\infty} f\left(u_{i}\right)
$$

where $f(x)=1+a_{1} x+a_{2} x^{2}+\ldots$ is a homogeneous series.

(b) Given an algebraic orientation on E, there is a canonical isomorphism

$$
E^{\star} M G L \cong E^{\star}\left[\left[b_{1}, \ldots, b_{n}, \ldots\right]\right] \subset E^{\star}\left[\left[x_{1}, \ldots, x_{k}, \ldots\right]\right],
$$

where $b_{i}$ are the elementary symmetric series in the $x_{i}$ 's. Moreover, under this isomorphism, maps of ring spectra $M G L \rightarrow E$ correspond to $\prod_{i=1}^{\infty} f\left(x_{i}\right)$, where $f$ as above.

Proof. To see (a), note that analogously to Proposition 2.6, we have

$$
E^{\star}(B G L \times B G L) \cong E^{\star}\left[\left[b_{i}, b_{i}^{\prime} \mid i=1,2, \ldots\right]\right],
$$

where $b_{i}$ are the elementary symmetric series in variables $u_{i}$. Now a ring spectra map $g \in E^{\star}\left[\left[b_{i}\right]\right]$ is seen to be characterized by

$$
g\left(u_{1}, u_{2}, \ldots\right) \cdot g\left(u_{1}^{\prime}, u_{2}^{\prime}, \ldots\right)=g\left(u_{1}, u_{1}^{\prime}, u_{2}, u_{2}^{\prime}, \ldots\right) .
$$

It is easily seen that such functions are of the form (2.15). (b) follows from (a) by the $E$-valued Thom isomorphism. 
The reader might be surprized why (s)he has not seen a formal group law on $E_{\star}$ yet. The reason is the same as in the Real case: even for a commutative associative ring spectrum $E$, the ring $E_{\star}$ is not necessarily (graded-) commutative. The reason is that the map $S^{2 \alpha} \rightarrow S^{2 \alpha}$ given by swapping the coordinates (which arises when commuting two elements $x, y$ of dimension $\alpha$ ) is homotopic in the stable category to the map $\iota: S^{\alpha} \rightarrow S^{\alpha}$ given by reversing the sign of the coordinate, which is not -1 !

In the Real case, $\pi_{0} S^{0}$ (we mean of course the stable group) is the Burnside ring of $\mathbb{Z} / 2$ (see $[13,18]$ ), and we have $\iota=[1]-[\mathbb{Z} / 2]$. In the algebraic case, $\pi_{0} S^{0}$ was studied by Fabien Morel [21], who conjectured that it is isomorphic to the Grothendieck-Witt ring of quadratic forms over $k$. In any case, if $k \subset \mathbb{R}$, the forgetful map to $\mathbb{Z} / 2$-equivariant spectra shows that $\iota \neq-1$ (which Morel proved in a different way through tensoring with $\mathbb{Q}$ ).

PROPOSITION 2.16. If $E$ is algebraically oriented, then $\iota=-1 \in \pi_{0} E$. Consequently, $E_{\star}$ is a graded-commutative ring, if we consider $k+\ell \alpha$ to have total degree $k+\ell$.

Proof. It suffices to consider $E=M G L$, since, by Proposition 2.14, there is a ring spectra map $M G L \rightarrow E$. The proof for $M G L$ is, actually, quite analogous to the Real case [13]. Recall that if $D$ is the prespectrum used to define $M G L$, then one has $D_{1+\alpha}=\left(\mathbb{P}^{\infty}\right)^{\gamma^{1}}=\mathbb{P}^{\infty}$. Since $\iota$ is represented (in the unstable category) by a map $S^{\alpha} \rightarrow S^{\alpha}$, it is represented by a map $S^{1+\alpha} \rightarrow D_{1+\alpha}=\mathbb{P}^{\infty}$. But recall that $\mathbb{P}^{\infty}=K(\mathbb{Z}, 1+\alpha)$, and so $\pi_{1+\alpha} \mathbb{P}^{\infty}=\mathbb{Z}$, with the isomorphism given by augmentation. Thus, $\iota=-1$ in this group.

COROLLARY 2.17. An algebraic orientation specifies a formal group law $F_{E}$ on $E_{\star}$.

Proof. As usual, just use the multiplication on $\mathbb{P}^{\infty} \simeq B \mathbb{G}_{m}$ (here by $B$ we mean the étale classifying space in the sense of Voevodsky).

Analogously to [13], Theorem 2.25, we have the following.

THEOREM 2.18. There is a bijective correspondence between:

(1) algebraic orientations on $E$,

(2) ring spectra maps $M G L \rightarrow E$,

(3) strict isomorphisms of formal group laws on $E_{\star}$ with source $F_{E}$.

Proof. Given an algebraic orientation $u$, Proposition 2.2 easily implies that an arbitrary algebraic orientation is of the form

$$
g(u)=u+a_{1} u^{2}+a_{2} u^{3}+\ldots
$$

This is an isomorphism of formal group laws. Thus, the correspondence between (1) and (3). 
Recalling Proposition 2.14 and substituting $f(u)=g(u) / u$ gives the correspondence between (1) and (2).

EXAMPLE. By the previous Theorem, localizing at $\ell$, we obtain the Quillen idempotent

$$
e: M G L_{(\ell)} \rightarrow M G L_{(\ell)},
$$

corresponding to the universal $\ell$-typical formal group law. We can set $B P G L=$ $e M G L_{(\ell)}$ where the right-hand side means localization at $\ell$.

Remark. We can also develop a theory of $E_{\infty}$-ring spectra and modules in the category of spaces over $k$, prove that $M G L$ is $E_{\infty}$, and then construct $B P G L$, as well as other spectra (such as Morava $K(n)$-theories) by killing appropriate sequences of the Lazard ring generators $x_{i}$. (For treatment of the relevant foundational issues, see Jardine [15] and also the first authors' paper [11].)

\section{Conjectures on $M G L$}

Since we have a formal group law on $M G L_{\star}$, we have a canonical map

$$
M U_{*} \rightarrow M G L_{\star}
$$

which sends an element of dimension $2 n$ to an element of dimension $n(1+\alpha)$. If $k \subseteq \mathbb{C}$, forgetting gives a map

$$
M G L_{\star} \rightarrow M U_{*}
$$

and it is easy to see that the composition of (3.2) and (3.1) is the identity, so $M U_{*}$ splits as a direct summand of $M G L_{\star}$ (additively).

Remark. It is quite likely that the above argument has an analogue for any field. For any field, one has another topological realization (forgetful) functor from $k$ spaces to spaces, namely the étale realization (see Artin-Mazur [4], p. 114). The étale realization can be extended to $k$-spaces in the usual way (similarly as the Real and complex realizations). It seems reasonable to conjecture that, up to completion at a prime different from the characteristic of the field, the étale realization of $M G L$ for $k$ algebraically closed is $M U_{*}\left[\sigma, \sigma^{-1}\right]$, where $\sigma$ is an element of dimension $1-\alpha$ (which expresses independence of twist).

Anyway, we are really interested in any additional information one might get about the maps (3.1),(3.2).

CONJECTURE 3.3. We have

$M G L^{k+\ell \alpha}(X)=0$ for a smooth variety $X$, and $k>\ell$,

The map (3.1) induces an iso $M G L_{*(1+\alpha)} \cong M U_{*}$. 
Remark. While this paper was under revision, Hopkins and Morel [9] announced a proof of this conjecture.

We refer to (3.4) as the transversality hypothesis, and to the entire conjecture as the strong transversality hypothesis. To explain this term, we use analogy with the Real case. There it is known that $M \mathbb{R}_{k+\ell \alpha}$ for $\ell>k$ (or $M R^{k+\ell \alpha}$ for $k>\ell$ ) agrees with the corresponding geometric Real cobordism group (which is the group of actual cobordism classes of Real manifolds) $[10,16]$. This can be interpreted as a type of transversality. An analogous (although perhaps somewhat loose) geometric interpretation to (3.4) can be given by noticing that if a similar type of transversality was true in the algebraic case, there would exist no algebraic varieties in the dimensions specified by (3.4)! (since, in fact, actual algebraic varieties would necessarily have dimensions $n(1+\alpha)$ ).

The next thing one may notice is that an analogue of (3.4), (3.5) is known (Voevodsky [27]) in the case when $M G L$ is replaced by $H$, ordinary motivic cohomology. Thus, one may ask if $M G L$ can be built up as a tower of copies of $H$, similarly as in the case of complex-oriented theories. We find it easier to phrase this conjecture for $B P G L$, after completing at a prime $\ell$ (we just set $\left.B P G L_{\ell}^{\wedge}=h o \lim \left(B P G L \wedge M \mathbb{Z} / \ell^{n}\right)\right)$.

\section{CONJECTURE 3.6.}

$$
B P G L_{\ell}^{\wedge} \simeq \text { ho } \lim _{\leftarrow} B_{i}
$$

such that there are fibrations of spectra

$$
\bigvee_{R:|R|=n} \Sigma^{\|R\|(1+\alpha)} H \mathbb{Z} / \ell \rightarrow B_{n} \rightarrow B_{n-1},
$$

where the summation is over all Milnor words $R=\left(r_{0}, r_{1}, \ldots\right)$ of length $n$, i.e. $r_{i}$ are non-negative intgers, all but finitely many of which are 0 , and length is defined by $|R|=\sum r_{i}$, and $\|R\|=\sum r_{i}\left(\ell^{i}-1\right)$.

We refer to this as the structure conjecture. Note that the analogue of the structure conjecture is true for ordinary topological $B P$ (see [6]), and also in the Real case (see [13]). Note also that the structure conjecture would immediately imply Voevodsky's conjecture [27] on the existence of a spectral sequence from $\bmod \ell$ motivic cohomology to algebraic Morava $K(n)$-theory.

Now note that in the Real case, we have a completion theorem with respect to Galois descent (the Galois group being $\mathbb{Z} / 2=\operatorname{Gal}(\mathbb{C} / \mathbb{R})$ ). Note also that completion theorems with respect to Galois descent are by definition true for étale realizations. Thus, a completion conjecture for a generalized cohomology theory in the category of $k$-spaces would be a Lichtenbaum-Quillen type conjecture. However, it is well known that even in the case of algebraic $K$-theory, a precise completion theorem with respect to Galois descent is false (for a simple explanation, see [20]). While a Lichtenbaum-Quilen type conjecture could be phrased for 
algebraic Morava $K(n)$-theories, in the case of $M G L$ and $B P G L$, where there are more non-trivial coefficient groups, this would be more difficult.

Note, however, [13], that in the Real case, the spectral sequence arising from the structure theorem for $B P \mathbb{R}$ (an Adams-type spectral sequence) gives essentially the same information as the spectral sequence arising from Galois descent. Thus, by analogy, we can interpret the structure conjecture for algebraic cobordism as a substitute for a completion conjecture with respect to Galois descent.

\section{Rost Motive, Hypersymplectic Morava $K(n)$-Theory and Hopf 1: the Toy Case}

In this section, we shall switch and work entirely in the category of $\mathbb{Z} / 2$-equivariant spectra. In the subsequent sections, we will be interested in developing analogues of some of the concepts introduced here in the algebraic case.

In the sequel, $M$ will denote a free Real manifold of dimension $\left(2^{n}-1\right)(1+\alpha)$ which represents $v_{n} \in M \mathbb{R}_{\star}$ (a Milnor manifold). As for any free manifold of non-equivariant dimension $2^{n+1}-2, \mathrm{CW}$ approximation gives a map

$$
\phi: M \rightarrow S\left(\left(2^{n+1}-1\right) \alpha\right)
$$

We know

LEMMA 4.2. There is an element

$$
\sigma^{-2^{n}} \in M \mathbb{R}^{2^{n}(1-\alpha)} S\left(\left(2^{n+1}-1\right) \alpha\right)
$$

which is a unit (invertible in the ring $M \mathbb{R}^{\star} S\left(\left(2^{n+1}-1\right) \alpha\right)$ ). Furthermore, as $M \mathbb{R}$ modules,

$$
K \mathbb{R}(n) \wedge S\left(\left(2^{n+1}-1\right) \alpha\right) \cong K \mathbb{R}(n)\left\{1, \sigma^{2^{n}}\right\} .
$$

Remark. We have $\Sigma^{2^{n+1}(1-\alpha)} K \mathbb{R}(n) \cong K \mathbb{R}(n)$ (cf. [13]). There is a filtration on $K \mathbb{R}(n)_{\star}$ such that

$$
E^{0} K \mathbb{R}(n)_{\star}=\mathbb{Z} / 2\left[v_{n}, v_{n}^{-1}\right][a] /\left(a^{2^{n+1}-1}\right)\left\{1, \sigma^{-1}, \ldots, \sigma^{-2^{n}+1}\right\} .
$$

Proof. The first statement is proved in [13]. We sketch the argument here for future use in Section 6 below. Consider $v_{n} \in \pi_{\left(2^{n}-1\right)(1+\alpha)} M \mathbb{R}$, given by a Milnor manifold. Then

$$
a^{2^{n+1}-1} v_{n}=0 \in M \mathbb{R}_{\star}
$$

for dimensional reasons (see [13]). Now consider the stable cofibration

$$
S\left(\left(2^{n+1}-1\right) \alpha\right)_{+} \rightarrow S^{0} \rightarrow S^{\left(2^{n+1}-1\right) \alpha} .
$$

Mapping into $M \mathbb{R}$, we obtain a cofibration

$$
F\left(S\left(\left(2^{n+1}-1\right) \alpha\right)_{+}, M \mathbb{R}\right) \longrightarrow \Sigma^{1-\left(2^{n+1}-1\right) \alpha} M \mathbb{R} \stackrel{a^{2^{n+1}-1}}{\longrightarrow} \Sigma M \mathbb{R} .
$$


Passing to homotopy, we have

$$
v_{n} \in \pi_{2^{n}(1-\alpha)} \Sigma^{1-\left(2^{n+1}-1\right) \alpha} M \mathbb{R}
$$

(since $\left.1-\left(2^{n+1}-1\right) \alpha+\left(2^{n}-1\right)(1+\alpha)=2^{n}(1-\alpha)\right)$. By (4.5), the element (4.8) lifts to the element (4.3). This element is a unit (=invertible), since its representative in the Borel cohomology spectral sequence is invertible ([13]).

Therefore, the collapse map $S\left(\left(2^{n+1}-1\right) \alpha\right)_{+} \rightarrow S^{0}$ induces a map

$$
K \mathbb{R}(n)\left\{1, \sigma^{2^{n}}\right\} \rightarrow F\left(S\left(\left(2^{n+1}-1\right) \alpha\right)\right)_{+}, K \mathbb{R}(n) .
$$

But examining non-equivariant Morava $K(n)$-theory, we see that this map induces an isomorphism on Borel (co)homology spectral sequences.

LEMMA 4.9. If $M$ is as above, then the collapse map

$$
M_{+} \wedge K \mathbb{R}(n) \rightarrow K \mathbb{R}(n)
$$

splits as a map of $M \mathbb{R}$-modules.

Proof. Let $v$ be the normal bundle of $M$ (in virtual dimension $-\left(2^{n}-1\right)(1+\alpha)$ ). The splitting is

$$
\begin{aligned}
& \Sigma^{\left(2^{n}-1\right)(1+\alpha)} K \mathbb{R}(n) \rightarrow \Sigma^{\left(2^{n}-1\right)(1+\alpha)} K \mathbb{R}(n) \wedge M^{v} \\
& \quad=\Sigma^{\left(2^{n}-1\right)(1+\alpha)} K \mathbb{R}(n) \wedge D\left(M_{+}\right) \cong K \mathbb{R}(n) \wedge M_{+} .
\end{aligned}
$$

By definition of a Milnor manifold, the composition of (4.10) and (4.11) is $v_{n}$.

LEMMA 4.12. If $M$ is as above, the map $\phi$ of (4.1) induces a map of $M \mathbb{R}$-modules

$$
M_{+} \wedge K \mathbb{R}(n) \rightarrow K \mathbb{R}(n) \vee \Sigma^{2^{n}(\alpha-1)} K \mathbb{R}(n),
$$

which splits as $M \mathbb{R}$-modules.

Proof. The first summand splits off by Lemma (4.9). The splitting on the second summand is the composition

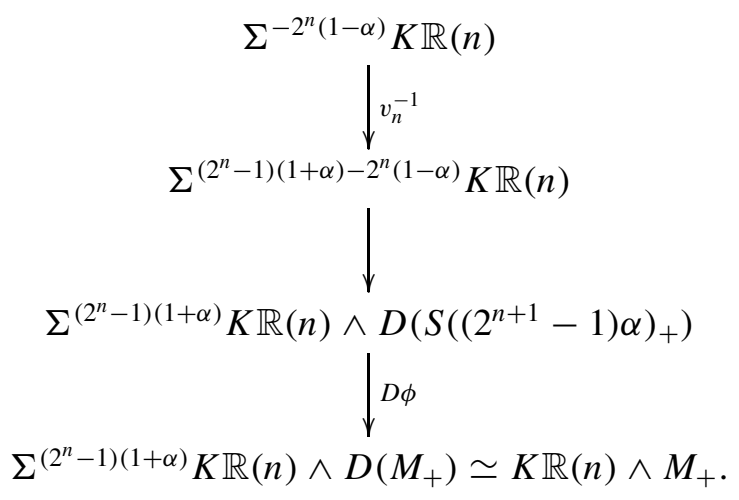


This is a splitting non-equivariantly, hence equivariantly by the Borel cohomology spectral sequence.

PROPOSITION 4.13. In the Borel cohomology spectral sequence

$$
H^{*}\left(\mathbb{Z} / 2, H^{*} M\right) \Rightarrow H^{*+\left(2^{n}-1\right) \alpha} M,
$$

consider

$$
0 \neq \iota \in H^{0}\left(\mathbb{Z} / 2, H^{\left(2^{n+1}-2\right)} M\right) .
$$

Then

$$
d_{2^{n+1}-1}(\iota) \neq 0 .
$$

Proof. Let $k(n)$ denote connective Morava $K$-theory. Then, non-equivariantly, the fundamental homology and unit cohomology classes and their canonical splittings induce a diagram

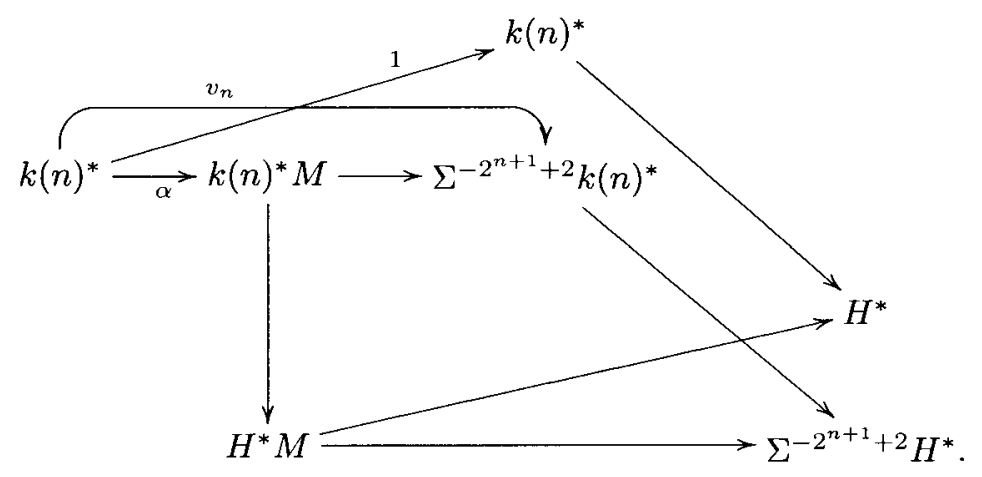

Furthermore, by the Atiyah-Hirzebruch spectral sequence, the

$$
k(n)^{*} \quad \text { and } \quad \Sigma^{-2^{n+1}+2} k(n)^{*}
$$

summands represent all of the Morava $k(n)$-cohomology of $M$ in dimensions $\equiv 0$ $\bmod 2^{n+1}-2$.

Now consider the Borel cohomology spectral sequence. By Lemma 4.12, when localizing the top part of the diagram by inverting $v_{n}$, in dimensions $\equiv 0 \bmod$ $2^{n+1}-2$ the spectral sequence will be a sum of one copy of the Borel cohomology spectral sequence for $K \mathbb{R}(n)$, and one copy of the Borel cohomology spectral sequence for $\Sigma^{2^{n}(1+\alpha)} K \mathbb{R}(n)$.

Now since the map $\alpha$ is induced by the collapse $M_{+} \rightarrow S^{0}$, the image of $\alpha$ is in the summand corresponding to the Borel cohomology spectral sequence for $K \mathbb{R}(n)$. Thus, $1 \oplus v_{n} \in k(n)^{*} \oplus \Sigma^{-2^{n+1}+2} k(n)^{*}$ is in this summand. Hence, both $1 \oplus 0,0 \oplus v_{n}$ project non-trivially to the $\Sigma^{2^{n}(1-\alpha)} K \mathbb{R}(n)$-summand. But these 
classes correspond to the generators of $H^{*}, \Sigma^{-2^{n+1}+2} H^{*}$. Thus, these generators are tied by any differentials occuring in the Borel cohomology spectral sequence for $\Sigma^{2^{n}(1-\alpha)} K \mathbb{R}(n)$. In particular, this applies to the differential in the statement of the Proposition.

THEOREM 4.14. Let $H$ be the $\mathbb{Z} / 2$-equivariant Eilenberg-MacLane spectrum associated with the constant Mackey functor $\mathbb{Z} / 2$. Let $\zeta \in H^{2^{n}+\left(2^{n}-1\right) \alpha} E \mathbb{Z} / 2_{+}$ be the non-trivial class. Consider the following fibration in the category of $\mathrm{H}$ modules:

$$
R \longrightarrow H \wedge E \mathbb{Z} / 2_{+} \stackrel{\zeta}{\longrightarrow} \Sigma^{2^{n}+\left(2^{n}-1\right) \alpha} H \wedge E \mathbb{Z} / 2_{+} .
$$

Then there exists a splitting of $H$-modules $R \rightarrow H \wedge M_{+} \rightarrow R$ such that the composition

$$
\Sigma^{\left(2^{n}-1\right)(1+\alpha)} H \wedge E \mathbb{Z} / 2_{+} \rightarrow R \rightarrow H \wedge M_{+}
$$

is the fundamental class, and the composition

$$
H \wedge M_{+} \rightarrow R \rightarrow H \wedge E \mathbb{Z} / 2_{+}
$$

is the unit class.

Proof. From Proposition 4.13 and Poincaré duality.

Remark. A version of the Theorem with coefficients $\mathbb{Z}$ is also easily obtained.

We need to mention one more construction, namely the $\mathbb{Z} / 2$-equivariant interpretation of the Hopf invariant 1 problem (see [13]). Note that the tangent bundle of the unit sphere in the sum of $2^{n}$ copies of the sign representation satisfies

$$
\tau_{S\left(2^{n} \alpha\right)} \oplus 1 \cong 2^{n} \alpha .
$$

Thus, if

$$
\tau_{S\left(2^{n} \alpha\right)} \cong 2^{n}-1
$$

then $\left(2^{n}\right)_{S\left(2^{n} \alpha\right)} \cong\left(2^{n} \alpha\right)_{S\left(2^{n} \alpha\right)}$ and hence in particular,

$$
\Sigma^{2^{n}(1-\alpha)} S\left(2^{n} \alpha\right) \simeq S\left(2^{n} \alpha\right) \text {. }
$$

Note that (4.15) is required to hold $\mathbb{Z} / 2$-equivariantly, which is a stronger formulation than the original Hopf invariant 1 problem. On the other hand, (4.16) is a stable statement, while the original Hopf 1 problem was unstable. At any rate, factoring (4.16) modulo 2 implies an equivalence of particular stunted projective spaces (cf. [8]), which turns out to happen for $n=1,2,3$, just as expected.

It is (4.16) that we will generalize to the algebraic case. 


\section{The Rost Space and Hypersymplectic Morava $K(n)$-Theories: the Algebraic Case}

In this section, we return to the world of Morel-Voevodsky's spaces over a field $k$, and resulting spectra category of spectra [28]. The algebraic analogue of Theorem 4.14 is Markus Rost's famous result on the Chow groups of quadrics [25]. The gadget $R$ became known as the Rost motive. The Rost motive played a crucial role in Voevodsky's proof of the Milnor conjecture.

The main idea of this paper is to ask the following questions:

(1) What is the algebraic analogue of $S\left(\left(2^{n+1}-1\right) \alpha\right)$, i.e. is there a space whose homology is the Rost motive?

(2) Is there an algebraic analogue of (4.4) and if yes, what is the algebraic analogue of $\Sigma^{2^{n}(1-\alpha)} K \mathbb{R}(n)$ ?

(3) Is there a $M G L$-module decomposition of $M G L \wedge X$ for a Pfister quadric $X$ analogous to the motivic decomposition constructed by Rost [25]?

(4) Is there an algebraic analogue of $S\left(2^{n} \alpha\right)$, and is there a meaningful algebraic analogue of the Hopf invariant 1 problem as stated at the end of last section? [23].

These questions are motivated by the following beautiful example of Quillen

EXAMPLE. Consider the projective quadratic curve $Q$ given by the equation

$$
a x^{2}+b y^{2}=z^{2}
$$

where $a, b \in k^{\times}$. If $K$ denotes the $\mathbb{P}^{1}$-spectrum of algebraic $K$-theory over the ground field $k$, then

$$
K \wedge Q_{+} \simeq K \vee K^{\perp}
$$

where $K^{\perp}$ is the algebraic $K$-theory of the central simple algebra $D$ associated with the symbol $(a, b) \in K_{2}^{M}(k)$. Explicitly, the spectrum $Z$ defining $K^{\perp}$ has $Z_{0}=$ $B G L(D) \times \mathbb{Z}$, and we have $\Omega^{1+\alpha} Z_{0} \simeq Z_{0}$. A map $K^{\perp} \rightarrow K \wedge Q_{+}$can be obtained from noticing that, under suitable interpretation, $Q$ has a 'non-commutative point' over $D$. Pursuing the analogy with the Real case, $Q$ is in this case the analogue of $S\left(\left(2^{2}-1\right) \alpha\right)$, and $K^{\perp}$ is the analogue of the Real symplectic $K$-theory, which is, by Real Bott periodicity, equivalent to $\Sigma^{2(1-\alpha)} K \mathbb{R}$.

The main goal of this section will be to find some generalization of Quillen's example to higher Morava $K$-theories, and thus answer questions 1,2 . Note that if $H$ denotes the spectrum of ordinary motivic cohomology with coefficients in $\mathbb{Z}$, then $Q \wedge H$ is the Rost motive of the quadratic curve $Q$.

CONSTRUCTION. Consider a Pfister form

$$
\left\langle\left\langle a_{1}, \ldots, a_{n}\right\rangle\right\rangle=\otimes\left(x^{2}-a_{i} y^{2}\right) .
$$


Let $X$ denote the projective quadric given by the equation

$$
\left\langle\left\langle a_{1}, \ldots, a_{n}\right\rangle\right\rangle=0 .
$$

Let, further, $Y$ denote the projective quadric given by the equation

$$
\left\langle\left\langle a_{1}, \ldots, a_{n}\right\rangle\right\rangle=z^{2} .
$$

Note that every Pfister form represents 1 (since it is true for $n=1$ ), and hence $Y$ is an isotropic quadric. Thus, picking a rational point $y \in Y$, we obtain a map

$$
Y_{+} \stackrel{\rho}{\longrightarrow} S^{\left(2^{n}-1\right)(1+\alpha)},
$$

(since the right-hand side is equivalent to $Y / Y-\{y\}$ ). Note that this does not depend on the choice of rational point, since an isotropic quadric is rational. On the other hand, we have the collapse map $Y_{+} \stackrel{\gamma}{\longrightarrow} S^{0}$. We define the Rost spectrum $\Theta$ by the cofibration

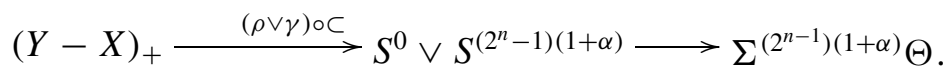

PROPOSITION 5.5. $H \wedge \Theta$ is the Rost motive.

Proof. Let, for the moment, $Y$ be the projective quadric given by the equation

$$
\left\langle\left\langle a_{1}, \ldots, a_{n}\right\rangle\right\rangle=b z^{2}
$$

for a general $b$. Markus Rost [25] proved that, if $R_{n-1}$ is the Rost motive associated with $\left(a_{1}, \ldots, a_{n}\right) \in K_{n}^{M}(k)$, and $R_{n}$ is the Rost motive associated with $\left(a_{1}, \ldots, a_{n}, b\right) \in K_{n+1}^{M}(k)$, then

$$
H \wedge Y_{+} \cong R_{n} \vee \bigvee_{k=1}^{2^{n-1}-1} \Sigma^{k(1+\alpha)} R_{n-1}
$$

and

$$
H \wedge X_{+} \cong \bigvee_{k=0}^{2^{n-1}-1} \Sigma^{k(1+\alpha)} R_{n-1},
$$

and, furthermore, the inclusion from $X$ to $Y$ induces the map from (5.7) to (5.6) which is the identity on

$$
\bigvee_{k=1}^{2^{n-1}-1} \Sigma^{k(1+\alpha)} R_{n-1}
$$

Applying Poincaré duality,

$$
H \wedge(Y /(Y-X)) \cong \bigvee_{k=1}^{2^{n-1}} \Sigma^{k(1+\alpha)} R_{n-1},
$$


and the map $Y_{+} \rightarrow Y /(Y-X)$, when smashed with $H$, induces the identity on (5.8). Now consider the cofibration of motives

$$
H \wedge(Y / Y-X) \longleftarrow v\left(v \wedge Y_{+} \longleftarrow u \wedge(Y-X)_{+} .\right.
$$

If we can construct a map $f$ from (5.8) to $H \wedge Y_{+}$which is an isomorphism between (5.8) and the same summands of $H \wedge Y_{+}$, then we can modify (5.10) as

$$
C(v \circ f) \leftarrow C f \leftarrow H \wedge\left(Y-X_{+}\right),
$$

and we see from the above that $C(v \circ f) \cong \Sigma^{2^{n-1}(1+\alpha)} R_{n-1}$, which is what we need to prove. But now if $b=1, Y$ is isotropic and hence, if we consider a fibration of spectra

$$
Z \stackrel{q}{\longrightarrow} Y_{+} \stackrel{\rho \vee \gamma}{\longrightarrow} S^{\left(2^{n-1}-1\right)(1+\alpha)} \vee S^{0},
$$

we can put $f=H \wedge q$ by the uniqueness of the splitting of the Rost motive (see [25], Proposition 13).

PROPOSITION 5.12. There exists a canonical cofibration

$$
S^{\left(2^{n-1}-1\right)(1+\alpha)} \rightarrow \Theta \rightarrow\left(S^{0}\right)^{\perp} .
$$

If $a_{1}=\cdots=a_{n}=1$ (the Pfister form is hyperbolic), then (5.13) is a split cofibration, and $\left(S^{0}\right)^{\perp}=S^{0}$.

Proof. Since $\left\langle\left\langle a_{1}, \ldots, a_{n}\right\rangle\right\rangle$ represents $1, Y-X$ has a rational point

$$
S^{0} \rightarrow(Y-X)_{+} .
$$

Thus, we can modify (5.4) as

$$
(Y-X)_{+} / S^{0} \rightarrow S^{\left(2^{n}-1\right)(1+\alpha)} \rightarrow \Sigma^{2^{n-1}(1+\alpha)} \Theta .
$$

Thus, we can put

$$
\left(S^{0}\right)^{\perp}=\Sigma^{1-2^{n-1}(1+\alpha)}\left(Y-X_{+} / S^{0}\right) .
$$

Now assume $a_{1}=\cdots=a_{n}=1$. Then $Y-X$ is isomorphic to the affine variety $Q$ given by the equation

$$
x_{1} y_{1}+\cdots+x_{k} y_{k}=1,
$$

where $k=2^{n-1}$. But note that we have a canonical bundle structure

$$
Q \rightarrow \mathbb{A}^{k}-\{0\}, \quad\left(x_{1}, \ldots, x_{k}, y_{1}, \ldots, y_{k}\right) \mapsto\left(y_{1}, \ldots y_{k}\right),
$$

with fiber $\mathbb{A}^{k-1}$. Thus, the map (5.16) is an equivalence. 
Thus, we wil be done with the second statement if we can show that

$$
Q=(Y-X) \rightarrow Y \rightarrow Y /(Y-\{y\})
$$

is homotopic to 0 (thus calculating the first map (5.4)). But to this end, it suffices to choose $y \in X$.

DEFINITION 5.17. With the same notation as in 5.12, put

$$
K(n-1)^{\perp}=K(n-1) \wedge\left(S^{0}\right)^{\perp} .
$$

Remark. While this is not displayed in the notation, $\left(S^{0}\right)^{\perp}, K(n-1)^{\perp}$ depend on the numbers $\left(a_{1}, \ldots, a_{n}\right)$ (as we can see already in the Quillen example for $n=2$ ). Moreover, if we do not assume the strong transversality hypothesis, $K(n-1)$ could depend on a particular choice of the Milnor variety we invert in $B P G L$. (Here by Milnor variety we mean any smooth projective variety of dimension $2^{n-1}-1$ over $k$ whose Segre number is $\ell$.) In this case, $\ell=2$ and we choose the Milnor variety which is the projective Pfister quadric given by the equation

$$
\left\langle\left\langle a_{1}, \ldots, a_{n-1}\right\rangle\right\rangle=a_{n} z^{2} .
$$

\section{Conjectural Splitting of the Morava $K(n)$-Theory of Quadrics Associated with Pfister Forms}

We next introduce an even more explicit model for the $M G L$-module $\Theta \wedge M G L$ associated with the Rost spectrum $\Theta$. Denote by $\left\langle\left\langle a_{1}, \ldots, a_{n}\right\rangle\right\rangle^{\prime}$ the pure Pfister form. Let $X^{\prime}$ be the projective quadric given by

$$
\left\langle\left\langle a_{1}, \ldots, a_{n}\right\rangle\right\rangle^{\prime}=0 .
$$

Then since

$$
\left\langle\left\langle a_{1}, \ldots, a_{n}\right\rangle\right\rangle-z^{2}
$$

contains as a direct summand a hyperbolic form, $Y$ contains a subvariety $Y^{\prime}$ which is a model for the Thom space of the canonical line bundle on $X^{\prime}: Y^{\prime}$ is given, say, by the equation $x_{0}-z=0$. Moreover, $Y / Y^{\prime} \simeq S^{\left(2^{n}-1\right)(1+\alpha)}$. On the other hand, we see that the composition $Y^{\prime} \rightarrow Y \rightarrow Y /(Y-X)$ is isomorphic to the $\gamma^{1}$-Thomification of the inclusion $X^{\prime} \subset X$.

We obtain the following.

\section{PROPOSITION 6.1.}

$$
\begin{aligned}
\Theta \wedge M G L & \simeq \Sigma^{\left(-2^{n-1}+1\right)(1+\alpha)} X / X^{\prime} \wedge M G L \\
& \simeq \Sigma^{\left(2^{n-1}-1\right)(1+\alpha)} F\left(\left(X-X^{\prime}\right)_{+}, M G L\right) .
\end{aligned}
$$


Further,

$$
D \Theta \wedge M G L \simeq \Sigma^{-\left(2^{n-1}-1\right)(1+\alpha)}\left(X-X^{\prime}\right)_{+} \wedge M G L .
$$

Proof. We just finished proving the first equality of the first statement. The other statements follow by Poincaré duality.

\section{COROLLARY 6.2.}

$$
\left(S^{0}\right)^{\perp} \wedge M G L \simeq \Sigma^{\left(2^{n-1}-1\right)(1+\alpha)+1} F\left(\widetilde{X-X^{\prime}}, M G L\right) .
$$

We now recall the first part of the proof of Lemma 4.2, and try to develop an algebraic analogue. We start with the cofibration sequence (4.6). In the algebraic world, we have a cofibration sequence

$$
\left(X-X^{\prime}\right)_{+} \rightarrow S^{0} \rightarrow \widetilde{X-X^{\prime}} .
$$

Mapping to $M G L$, we get

$$
F\left(\widetilde{X-X^{\prime}}, M G L\right) \rightarrow M G L \rightarrow F\left(\left(X-X^{\prime}\right)_{+}, M G L\right),
$$

or

$$
\Sigma^{-1-\left(2^{n-1}-1\right)(1+\alpha)}\left(S^{0}\right)^{\perp} \wedge M G L \rightarrow M G L \rightarrow F\left(\left(X-X^{\prime}\right)_{+}, M G L\right),
$$

or

$$
F\left(\left(X-X^{\prime}\right)_{+}, M G L\right) \stackrel{\delta}{\longrightarrow} \Sigma^{\left(2^{n-1}-1\right)(1+\alpha)}\left(S^{0}\right)^{\perp} \wedge M G L \stackrel{\gamma}{\longrightarrow} \Sigma M G L .
$$

Now we can consider

$$
v_{n-1} \in\left[\left(S^{0}\right)^{\perp}, \Sigma^{\left(2^{n-1}-1\right)(1+\alpha)}\left(S^{0}\right)^{\perp} \wedge M G L\right] .
$$

We would like to conjecture that $v_{n-1}$ is sent to 0 by $\gamma$, and lifts to a unit element in the homotopy of the left-hand side of (6.5), but in order for that to make sense, we must first address the issue in what sense $\left(S^{0}\right)^{\perp}$ is a 'degree' in the stable homotopy groups of $M G L$. We combine all this in the following.

CONJECTURE 6.7 (The periodicity conjecture).

(a) $\left(S^{0}\right)^{\perp} \wedge D\left(S^{0}\right)^{\perp} \wedge M G L \simeq M G L$.

(b) The element $v_{n-1}$ written as in (6.6) satisfies $\gamma\left(v_{n-1}\right)=0$ in (6.5), and lifts by $\delta$ to a unit (invertible) element $u$ in the stable homotopy of $F\left(\left(X-X^{\prime}\right)_{+}, M G L\right)$, where $\left(S^{0}\right)^{\perp}$ is considered a 'dimension' in stable homotopy of $M G L$-modules by (a). 
PROPOSITION 6.8. Assuming the periodicity conjecture, we have

$\Theta \wedge M G L^{\perp} \simeq \Theta \wedge M G L$,

$D \Theta \wedge M G L^{\perp} \simeq D \Theta \wedge M G L$.

Proof. Assuming the conjecture, cup product with $u$ defines an equivalence

$$
F\left(\left(X-X^{\prime}\right)_{+}, M G L\right) \longrightarrow F\left(\left(S^{0}\right)^{\perp} \wedge\left(X-X^{\prime}\right)_{+}, M G L\right),
$$

while cap product with $u$ defines an equivalence

$$
\left(S^{0}\right)^{\perp} \wedge\left(X-X^{\prime}\right)_{+} \wedge M G L \simeq\left(X-X^{\prime}\right)_{+} \wedge M G L .
$$

The statement now follows from Proposition 6.1.

Now for any space $M$, let $E M$ be the Čech resolution of $M$. For any spectrum $E$, we can define the $M$-Tate spectrum $\hat{E}_{M}=\widetilde{E M} \wedge F\left(E M_{+}, E\right)$ (recall that " , denotes unreduced suspension).

LEMMA 6.9. Let $M$ be a Milnor variety representing an element $v_{n} \in B P G L$. Then $K(n)$ constructed by inverting $v_{n}$ is $M$-complete in the sense that

$$
E M_{+} \wedge K(n) \stackrel{\simeq}{\longrightarrow} K(n) \longrightarrow \stackrel{\simeq}{\longrightarrow} F\left(E M_{+}, K(n)\right),
$$

and

$$
\widehat{K(n)}_{M} \simeq * .
$$

Proof. The first and second equivalence of (6.10) clearly imply (6.11), since we have a cofibration

$$
E M_{+} \wedge E \stackrel{N}{\longrightarrow} F\left(E M_{+}, E\right) \longrightarrow \hat{E}_{M}
$$

where $N$ is the composition of the two maps (6.10). This is because

$$
E M_{+} \wedge F\left(E M_{+}, E\right) \underset{\phi}{\simeq} E M_{+} \wedge E .
$$

To see (6.12), the map displayed is induced by the collapse $E M_{+} \rightarrow S^{0}$. A map the other way is

$$
E M_{+} \wedge F\left(E M_{+}, E\right) \underset{\psi}{\stackrel{\Delta \wedge I d}{\longrightarrow} E M_{+} \wedge E M_{+} \wedge} \begin{array}{r}
\wedge \\
\downarrow \\
\qquad M_{+} \wedge\left(E M_{+}, E\right)
\end{array}
$$


The composition $\psi \phi$ is:

$$
\begin{aligned}
& E M_{+} \wedge E \stackrel{\Delta \wedge I d}{\longrightarrow} E M_{+} \wedge E M_{+} \wedge E \stackrel{I d \wedge \epsilon \wedge I d}{\longrightarrow} E M_{+} \wedge E M_{+} \wedge E \\
& \underset{ }{\downarrow} M_{+} \wedge E,
\end{aligned}
$$

which is the identity. The composition $\phi \psi$ is:

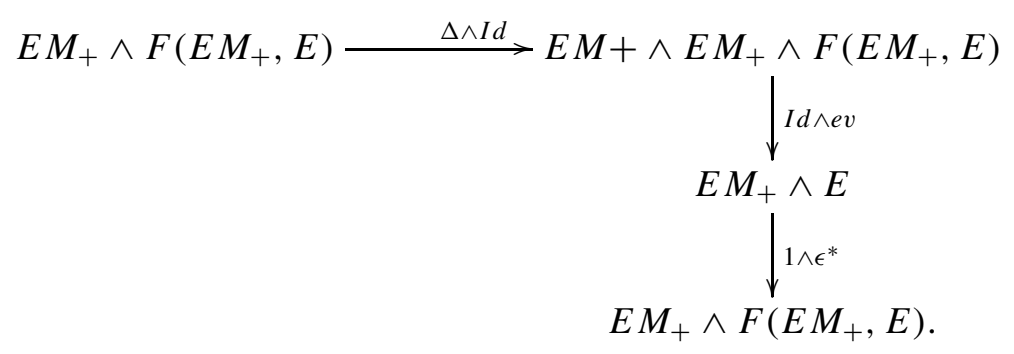

We see that (6.13) is homotopic to the identity via the standard simplicial homotopy between the two projections

$$
\begin{aligned}
E M & \times E M \rightarrow E M: h_{u}\left(\left(m_{0}, \ldots, m_{k}\right)\left[s_{0}, \ldots, s_{k}\right] \times\right. \\
& \left.\times\left(n_{0}, \ldots, n_{\ell}\right)\left[t_{0}, \ldots, t_{\ell}\right]\right)=\left(m_{0}, \ldots, m_{k}, n_{0}, \ldots, n_{\ell}\right) \times \\
& \times\left[e s_{0}, \ldots, u s_{k},(1-u) t_{0}, \ldots,(1-u) t_{\ell}\right] .
\end{aligned}
$$

Thus, it suffices to prove (6.10).

For the first equivalence of (6.10), we have an equivalence $M \times E M \rightarrow M$ (given by the projection), so we have a projection equivalence

$$
K(n) \wedge M_{+} \wedge E M_{+} \rightarrow K(n) \wedge M_{+} .
$$

Thus, it suffices to show that

$$
K(n) \text { is a wedge summand of } K(n) \wedge M_{+} .
$$

To see (6.14), consider the composition

$$
M^{v} \rightarrow M^{v} \wedge M_{+} \rightarrow M G L \wedge M_{+},
$$

(where $v$ is the virtual normal bundle set in dimension 0 ) which induces an equivalence of $M G L$-modules

$$
K(n) \wedge M^{v} \stackrel{\simeq}{\longrightarrow} K(n) \wedge M_{+} .
$$

Now the canonical maps

$$
S^{\left(2^{n}-1\right)(1+\alpha)} \rightarrow M^{v}, \quad M_{+} \rightarrow S^{0}
$$


give a composition

$$
\Sigma^{\left(2^{n}-1\right)(1+\alpha)} K(n) \rightarrow K(n) \wedge M^{v} \simeq K(n) \wedge M_{+} \rightarrow K(n),
$$

which, by definition, is $v_{n}$ as a map of $M G L$-modules. This is the splitting (6.14), and hence the first equivalence (6.10).

Similarly, to get the second equivalence (6.10), we have, of course, an equivalence

$$
F\left(E M_{+} \wedge M_{+}, K(n)\right) \stackrel{\simeq}{\longleftarrow} F\left(M_{+}, K(n)\right) .
$$

Thus, to prove the second equivalence (6.10), it suffices to prove

$$
K(n) \text { is a direct summand of } F\left(M_{+}, K(n)\right) .
$$

But similarly as above, the maps (6.15) induce a composition

$$
\Sigma^{-\left(2^{n}-1\right)(1+\alpha)} K(n) \leftarrow F\left(M^{v}, K(n)\right) \simeq F\left(M_{+}, K(n)\right) \leftarrow K(n),
$$

which is, again, $v_{n}$ by definition of a Milnor variety. Thus, the splitting proves (6.16), and hence the second equivalence of (6.10).

In the proof, we obtained in particular the following.

COROLLARY 6.17. If $M$ is a smooth projective variety representing the element $v_{n} \in B P G L_{\star}$, then the canonical maps

$$
K(n) \wedge M_{+} \rightarrow K(n)
$$

and

$$
K(n) \rightarrow F\left(M_{+}, K(n)\right)
$$

are the projection (resp. injection) to (resp. from) a wedge summand.

THEOREM 6.18. Assuming the transversality hypothesis and the periodicity conjecture, we have

$$
\begin{aligned}
& \Theta \wedge K(n-1) \simeq K(n-1) \vee K(n-1)^{\perp} \simeq D \Theta \wedge K(n-1), \\
& \quad K(n-1)^{\perp} \wedge_{M G L} M G L^{\perp} \simeq K(n-1) .
\end{aligned}
$$

Proof. Let $Q$ be the Pfister (projective) quadric given by

$$
\left\langle\left\langle a_{1}, \ldots, a_{n-1}\right\rangle\right\rangle=a_{n} y^{2} .
$$

By the above argument, we have also

$$
Q_{+} \wedge M G L^{\perp} \simeq Q_{+} \wedge M G L .
$$


Now take the cofibration

$$
\Sigma^{\left(2^{n-1}-1\right)(1+\alpha)} M G L \rightarrow \Theta \wedge M G L \rightarrow M G L^{\perp} .
$$

Smashing with $Q_{+}$and applying (6.19), we get a cofibration of $M G L$-modules

$$
\Sigma^{\left(2^{n-1}-1\right)(1+\alpha)} M G L \wedge Q_{+} \stackrel{\alpha}{\longrightarrow} Q_{+} \wedge \Theta \wedge M G L \stackrel{\beta}{\longrightarrow} Q_{+} \wedge M G L .
$$

But by the transversality hypothesis, the connecting map of (6.21) is 0 . Thus,

$$
Q_{+} \wedge \Theta \wedge M G L \simeq\left(Q_{+} \wedge M G L\right) \vee\left(\Sigma^{\left(2^{n-1}-1\right)(1+\alpha)} Q_{+} \wedge M G L\right)
$$

and hence

$$
Q_{+} \wedge \Theta \wedge K(n-1) \simeq\left(Q_{+} \wedge K(n-1)\right) \vee\left(Q_{+} \wedge K(n-1)\right)
$$

Considering the collapse map

$$
Q_{+} \wedge \Theta \wedge K(n-1) \rightarrow \Theta \wedge K(n-1)
$$

one summand (6.23) maps by definition trivially, the other summand produces a map which, over a splitting field, is a wedge summand projection from the top and bottom class of $Q$.

Thus, since $Q$ is a Milnor variety, the composition

$$
\begin{aligned}
& K(n-1)^{\perp} \longleftarrow \Theta \wedge K(n-1) \longleftarrow \gamma \quad Q_{+} \wedge K(n-1) \\
& \uparrow D \gamma \\
& D \Theta \wedge K(n-1)=D \Theta \wedge K(n-1)^{\perp} \\
& \uparrow \\
& K(n-1)^{\perp}
\end{aligned}
$$

is $v_{n-1}$, hence an isomorphism. Hence, the cofibration

$$
K(n-1) \rightarrow \Theta \wedge K(n-1) \rightarrow K(n-1)^{\perp}
$$

splits (the above composition provides a splitting). This concludes the proof of the first statement. 
Now for the second statement, compute

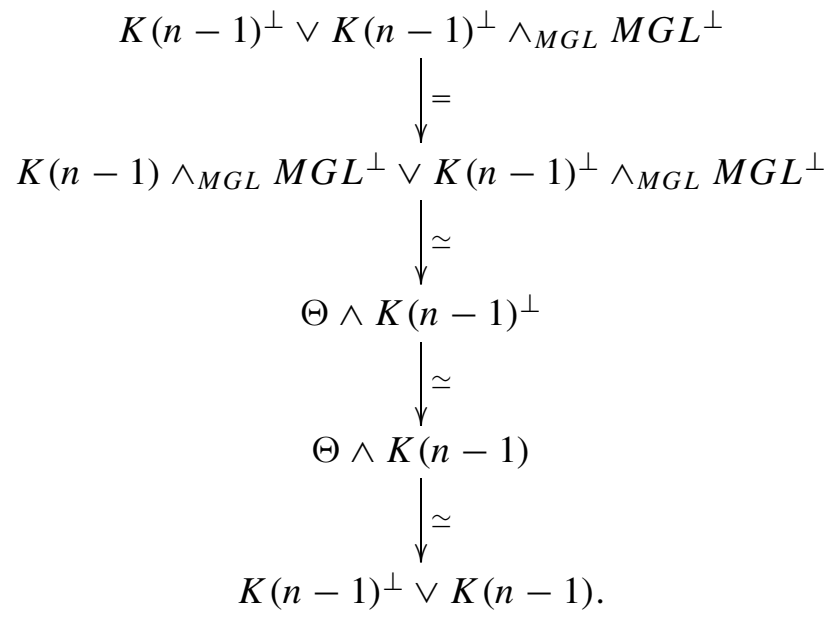

THEOREM 6.24. Assuming the transversality hypothesis and the periodicity conjecture,

$$
X_{+} \wedge K(n-1) \simeq \bigvee_{k=0}^{2^{n-1}-1} \Sigma^{k(1+\alpha)}\left(K(n-1) \vee K(n-1)^{\perp}\right)
$$

Proof. By Theorem 6.18, we have a map

$$
\begin{gathered}
\Sigma^{\left(2^{n-1}-1\right)(1+\alpha)} K(n-1) \vee K(n-1)^{\perp} \\
=D \Theta \wedge K(n-1)
\end{gathered}
$$

Now compose (6.25) with

$$
K_{+} \wedge K(n-1) \stackrel{\text { collapse }}{\longrightarrow} K(n-1) .
$$

We see that

Over a splitting field, the composition of (6.26) and (6.25)

is 0 on the second summand, and 1 on the first summand.

Applying $\wedge_{M G L} M G L^{\perp}$, however, we see that the composition

$$
\begin{gathered}
K(n-1) \vee K(n-1)^{\perp} \longrightarrow D \Theta \wedge K(n-1) \simeq D \Theta \wedge K(n-1)^{\perp} \\
X_{+} \wedge K(n-1)^{\perp} \\
\quad \text { collapse } \\
K(n-1)^{\perp}
\end{gathered}
$$


is an equivalence on the second summand. Combining (6.27) and (6.28), we get a composition

$$
K(n-1) \vee K(n-1)^{\perp} \longrightarrow X_{+} \wedge K(n-1) \stackrel{\beta}{\longrightarrow} K(n-1) \vee K(n-1)^{\perp}
$$

which is an iso over a splitting field, and hence an iso by the completion theorem for $K(n-1)$. Now consider the map

$$
\begin{gathered}
X_{+} \wedge K(n-1) \stackrel{\Delta \wedge 1}{\longrightarrow} X_{+} \wedge X_{+} \wedge K(n-1) \\
X_{+} \wedge K(n-1) \wedge X_{+} \\
\left(K(n-1) \vee K(n-1)^{\perp}\right) \wedge X_{+} \\
\quad I d \wedge I d \wedge c \\
\left(K(n-1) \vee K(n-1)^{\perp}\right) \wedge \mathbb{P}_{+}^{2^{n}-1} \\
\quad \simeq \\
\bigvee_{k=0}^{2^{n}-1} \Sigma^{k(1+\alpha)}\left(K(n-1) \vee K(n-1)^{\perp}\right) \\
\quad \mid \text { projection } \\
\bigvee_{k=0}^{2^{n-1}-1} \Sigma^{k(1+\alpha)}\left(K(n-1) \vee K(n-1)^{\perp}\right) .
\end{gathered}
$$

Over a splitting field, we see easily that this is an equivalence, hence it is an equivalence by the completion theorem for $K(n-1)$.

Remark. It is interesting to ask if $S^{\perp}$, or, more generally, an unreduced suspension of an affine quadric $\langle\phi\rangle=1$, is invertible under the smash product.

Recall from equivariant stable homotopy theory that with a compact Lie group $G$, we can associate the Burnside ring $A(G)$, a representation ring $R O(G)$ and a group of invertible $G$-spectra which we will for the moment denote by $\operatorname{Pic}(G-$ spectra $)$. There are canonical homomorphisms of groups

$$
R O(G) \rightarrow \operatorname{Pic}(G-\text { spectra }) \rightarrow A(G)^{\times} .
$$

The second map is the Euler characteristic. $G$-equivariant cohomology theories are indexed by $R O(G)$, but obviously the value of a cohomology theory only depends in the image of the dimension in $\operatorname{Pic}(G-$ spectra $)$.

The function of the Burnside ring in equivariant stable homotopy theory is that it is equal to

$$
\pi_{0} S^{0}
$$


Thus, we may define

$$
A_{\mathrm{Mot}}=\pi_{0} S^{0},
$$

$\mathrm{Pic}_{\mathrm{Mot}}=\{$ The group of $k$-spectra invertible under the smash product $\}$.

In other words, while we are used to indexing (generalized) motivic cohomology by $k+\ell \alpha$, to give full information, the theories should, in fact, be indexed by $\mu \in \mathrm{Pic}_{\mathrm{Mot}}$. Of course, it would also be interesting to know if there is a ring which would play the role of $R O_{\mathrm{Mot}}$.

Morel [21] defined a map from the Grothendieck-Witt ring $G W$ to $A_{\mathrm{Mot}}$, and conjectured that it is iso.

We do not really know what $\mathrm{Pic}_{\mathrm{Mot}}$ is, but we have one non-trivial example [12] of elements of Pic $\mathrm{Mot}_{\text {. }}$ Recall the following standard construction.

DEFINITION 6.31. The join $X * Y$ of two spaces $X, Y$ is defined as the homotopy pushout of the diagram<smiles>[Y][Y][V]</smiles>

It is well known that the join is commutative and associative.

LEMMA 6.32. Let $X$ be any space. Denote by $\tilde{X}$ the cofiber $X_{+} \rightarrow S^{0} \rightarrow \tilde{X}$ (the unreduced suspension of $X$ ). Then $\widetilde{X * Y}=\tilde{X} \wedge \tilde{Y}$. Furthermore, if $X$ is based, then $X * Y=X \wedge \tilde{Y}$.

Now let $X=\operatorname{Spec}\left(k[x] /\left(x^{2}-a\right)\right)$. Let $U$ be the affine quadric given by $x^{2}-a y^{2}=1$.

PROPOSITION 6.33. [12]

$$
\tilde{X} \wedge U \simeq \mathbb{P}^{1} \text {. }
$$

Proof. Let $Y$ be the projective quadric given by $x^{2}-a y^{2}=z^{2}$. We have $Y \cong \mathbb{P}^{1}$. Now if $\sqrt{a} \in k$, our statement asserts just that $\mathbb{G}_{m} \wedge S^{1} \simeq \mathbb{P}^{1}$, which is true. Thus, assume $\sqrt{a} \notin k$. Then $X=\operatorname{Spec}(L)$ where $L=k[\sqrt{a}]$. Further, $U=Y-\operatorname{Spec}(L)$. Now consider the étale map

$$
\operatorname{Spec}(L) \times \mathbb{A}^{1} \rightarrow X
$$

given by the isomorphism $\{\sqrt{a}\} \times \mathbb{A}^{1} \cong X-\{\sqrt{a}\}$ over $L$. By the colimit axiom with respect to coverings in the Nisnievich topology, we have a pushout of spaces

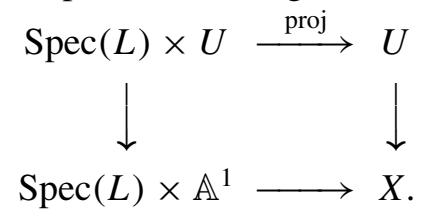

Thus, $\mathbb{P}^{1} \simeq \operatorname{Spec}(L) * U$. But, since $U$ is based, we have $\mathbb{P}^{1} \simeq \widetilde{\operatorname{Spec}(L)} \wedge U$. 


\section{Non-Associative Division Algebras and the Algebraic Hopf 1 Property}

DEFINITION 7.1. Let $k$ be a field, let $V$ be a vector space over $k$ with a quadratic form $Q$. A non-associative division algebra with norm $Q$ consists of the following data:

(1) a multiplication $V \otimes_{k} V \rightarrow V$

(2) a unit $1: k \rightarrow V$

(3) a conjugation "-": $V \rightarrow V$ (which is a linear involution)

such that the multiplication is unital, and

$$
\begin{aligned}
& (x y) \bar{y}=x Q(y), \\
& \overline{x y}=\bar{y} \bar{x} .
\end{aligned}
$$

THEOREM 7.4. Suppose $Q=\left\langle\left\langle a_{1}, \ldots, a_{n}\right\rangle\right\rangle$. Then for $n \leqslant 3$, there exists a nonassociative division algebra with norm $Q$.

Proof. If $n=1$, just take $C=k[i] /\left(i^{2}-a_{1}\right)$. If $n=2$, take the quaternion algebra $H$ associated with the symbol $\left(a_{1}, a_{2}\right)$ : It is generated as an associative algebra by two elements $i, j$ with the relations

$$
i^{2}=a_{1}, \quad j^{2}=a_{2}, \quad i j=-j i .
$$

We have, for $x, y \in C, \overline{x+j y}=\bar{x}-j$. We have, for $u, v \in C$ (recall that $u j=j \bar{u}$ )

$$
\begin{aligned}
(u+j v)(\overline{u+j v}) & =(u+j v)(\bar{u}-j v) \\
& =u \bar{u}+a_{2} v \bar{v}+j v \bar{u}-u j v=u \bar{u}+a_{2} v \bar{v},
\end{aligned}
$$

as claimed. Now for $n=3$, let $O$ consist of all pairs $u+\ell v, u, v \in H$ with the following multiplication

$$
(u+\ell v)(x+\ell y)=u x-a_{3} y \bar{v}+\ell(\bar{u} y+x v)
$$

and conjugation

$$
\overline{u+\ell v}=\bar{u}-\ell v \text {. }
$$

To verify (7.2), compute

$$
\begin{aligned}
& ((u+\ell v)(x+\ell y))(\bar{x}-\ell y) \\
& \quad=\left(u x-a_{3} y \bar{v}+\ell(\bar{u} y+x v)\right)(\bar{x}-\ell y)=u x \bar{x}-a_{3} y \overline{v x}+a_{3} y \bar{y} u+ \\
& \quad+a_{3} y \bar{v} \bar{x}+\ell\left(-\overline{x u} y+a_{3} v \bar{y} y+\bar{x} x v+\overline{x y} y\right)=(u+\ell v)\left(x \bar{x}+a_{3} y \bar{y}\right),
\end{aligned}
$$

as required.

To verify (7.3), compute

$$
(\bar{x}-\ell y)(\bar{u}-\ell v)=\overline{x u}-a_{3} v \bar{y}+\ell(-x v-\bar{u} y)=\overline{(u+\ell v)(x+\ell y)} .
$$


Remark. The term 'non-associative division algebra' first of all, of course, means 'not necessarily associative' (it could happen to be associative). But also, it only has the expected meaning if $Q$ is non-isotropic (in other words, if $Q$ is isotropic, the algebra can have divisors of 0 ).

LEMMA 7.5. $Q(x y)=Q(x) Q(y)$ or $Q(x)=0$. (In particular, the first case always occurs if $Q$ is unisotropic.)

Proof. First notice that

$$
\bar{x}(x y)=\overline{(\overline{x y}) x}=\overline{(\overline{y x}) x}=\overline{\bar{y}} Q(x)=Q(x) y .
$$

Next,

$$
(\bar{u}(u v)) \bar{v}=Q(u) v \bar{v}=Q(u) Q(v)=\bar{u}((u v) \bar{v}) .
$$

Now set

$$
u=\bar{x}, \quad v=x y .
$$

Then

$$
\bar{u}=x, \quad u v=\bar{x}(x y)=y Q(x) \quad \text { by (7.6). }
$$

So,

$$
\begin{aligned}
Q(x) Q(x y) & =(\bar{u}(u v)) \bar{v}=\text { by }(7.7) \\
\bar{u}((u v) \bar{v}) & =Q(x) x(y(\overline{x y}))=Q(x) x(y(\bar{y} \bar{x}))=Q(x)^{2} Q(y) .
\end{aligned}
$$

Remark. By Lemma 7.5, a non-associative division algebra with unisotropic norm $Q$ is in particular a composition algebra in the sense of Rost [26]. A wellknown theorem of Hurwitz [14] asserts that the only possible dimensions of composition algebras over fields of characteristic $\neq 2$ are $1,2,4$ and 8 , which gives a partial converse to Theorem 7.4.

CONVENTION. For a Pfister form $\left\langle\left\langle a_{1}, \ldots, a_{n}\right\rangle\right\rangle$, let $U_{n}$ denote the affine Pfister quadric

$$
\left\langle\left\langle a_{1}, \ldots, a_{n-1}\right\rangle\right\rangle=a_{n},
$$

and let $X_{n}$ denote the projective quadric $\left\langle\left\langle a_{1}, \ldots, a_{n}\right\rangle\right\rangle=0$.

Note: $U_{n}$ is the algebraic analogue of $S\left(2^{n-1} \alpha\right)$.

Let $H_{k}$ be the $k$-dimensional hyperbolic form. Then let $U_{n}^{0}$ be the affine quadric

$$
\left\langle\left\langle a_{1}, \ldots, a_{n-1}\right\rangle\right\rangle=1,
$$

let $X_{n}^{0}$ be the projective quadric $H_{2^{n}}=0$. Let $Y_{n}$ be the projective quadric $\left\langle\left\langle a_{1}, \ldots, a_{n}\right\rangle\right\rangle=z^{2}$.

Let $Y_{n}^{0}$ be the same quadric with $\left\langle\left\langle a_{1}, \ldots, a_{n}\right\rangle\right\rangle$ replaced by the hyperbolic form. 
THEOREM 7.8. Suppose there exists a non-associative division algebra with norm $\left\langle\left\langle a_{1}, \ldots, a_{n-1}\right\rangle\right\rangle$. Then we have isomorphisms of varieties over $k$

$$
\begin{aligned}
& U_{n} \times U_{n} \cong U_{n} \times U_{n}^{0}, \\
& U_{n} \times X_{n} \cong U_{n} \times X_{n}^{0}, \\
& U_{n} \times Y_{n} \cong U_{n} \times Y_{n}^{0}, \\
& U_{n} \times\left(Y_{n}-X_{n}\right) \cong U_{n} \times\left(Y_{n}^{0}-X_{n}^{0}\right) .
\end{aligned}
$$

Proof. We will give the proof of (7.9) and (7.10). The proofs of (7.11) and (7.12) are analogous.

For (7.9), let

$$
\left(x_{1}, \ldots, x_{2^{n-1}}, y_{1}, \ldots, y_{2^{n-1}}\right) \in U_{n} \times U_{n} .
$$

Then, setting $Q=\left\langle\left\langle a_{1}, \ldots, a_{n-1}\right\rangle\right\rangle$,

$$
Q\left(x_{1}, \ldots, x_{2^{n-1}}\right)=Q\left(y_{1}, \ldots, y_{2^{n-1}}\right)=a_{n} .
$$

So,

$$
Q\left(\frac{1}{a_{n}} \overline{\left(x_{1}, \ldots, x_{2^{n-1}}\right)}\left(y_{1}, \ldots, y_{2^{n-1}}\right)\right)=1 .
$$

Hence, we can send (7.13) to

$$
\left(x_{1}, \ldots, x_{2^{n-1}}, \frac{1}{a_{n}} \overline{\left(x_{1}, \ldots, x_{2^{n-1}}\right)}\left(y_{1}, \ldots, y_{2^{n-1}}\right)\right) \in U_{n} \times U_{n}^{0} .
$$

Obviously, this is an isomorphism.

For (7.10), let

$$
\left(x_{1}, \ldots, x_{2^{n-1}}, y_{1}, \ldots, y_{2^{n}}\right) \in U_{n} \times X_{n} .
$$

Then

$$
Q\left(x_{1}, \ldots, x_{2^{n-1}}\right)=a_{n}, Q\left(y_{1}, \ldots, y_{2^{n-1}}\right)=a_{n} Q\left(y_{2^{n-1}+1}, \ldots, y_{2^{n}}\right) .
$$

So,

$$
Q\left(y_{1}, \ldots, y_{2^{n-1}}\right)=Q\left(\frac{1}{a_{n}} \overline{\left(x_{1}, \ldots, x_{2^{n-1}}\right)}\left(y_{2^{n-1}+1}, \ldots, y_{2^{n}}\right)\right) .
$$

Note that the projective quadric

$$
Q\left(y_{1}, \ldots, y_{2^{n-1}}\right)=Q\left(z_{1}, \ldots, z_{2^{n-1}}\right)
$$

is isomorphic to $X_{n}^{0}$. Now we can send (7.14) to

$$
\left(x_{1}, \ldots, x_{2^{n-1}}, y_{1}, \ldots, y_{2^{n-1}}, \frac{1}{a_{n}} \overline{\left(x_{1}, \ldots, x_{2^{n-1}}\right)}\left(y_{2^{n-1}+1}, \ldots, y_{2^{n}}\right)\right) \in U_{n} \times Z_{n}^{0} .
$$


We now formulate the algebraic Hopf invariant one property as an analogue of (1.8), and prove its connection with non-associative division algebras.

DEFINITION 7.15. We say that $\left\langle\left\langle a_{1}, \ldots, a_{n}\right\rangle\right\rangle$ has the algebraic Hopf invariant 1 property if

$$
\left(U_{n}\right)_{+} \simeq\left(U_{n}\right)_{+} \wedge\left(S^{0}\right)^{\perp}
$$

in the stable category.

Remark. Note that the periodicity conjecture 6.7 implies that, analogously with the Real-oriented case, the algebraic Hopf invariant 1 property always holds in the category of $M G L$-modules, i.e. we have

$$
\left(U_{n}\right)_{+} \wedge M G L \simeq\left(U_{n}\right)_{+} \wedge\left(S^{0}\right)^{\perp} \wedge M G L .
$$

To see this, recall that $X-X^{\prime}$ was an affine variety given by the equation

$$
\left\langle\left\langle a_{1}, \ldots, a_{n}\right\rangle\right\rangle^{\prime}=-1 .
$$

This can be rewritten as

$$
\left\langle\left\langle a_{1}, \ldots, a_{n-1}\right\rangle\right\rangle^{\prime}-a_{n}\left\langle\left\langle a_{1}, \ldots, a_{n-1}\right\rangle\right\rangle=-1
$$

(where we take different sets of variables in the two Pfister forms indicated). In particular, (7.18) follows from

$$
a_{n}\left\langle\left\langle a_{1}, \ldots, a_{n-1}\right\rangle\right\rangle=1,
$$

which is equivalent to the equation of $U_{n}$. Thus, we have an embedding $U_{n} \subset$ $X-X^{\prime}$. Thus, assuming the periodicity conjecture, we can pull back the unit element $u \in\left[\left(S^{0}\right)^{\perp}, F\left(\left(X-X^{\prime}\right)_{+}, M G L\right)\right]$ to a unit (invertible) element

$$
w \in\left[\left(S^{0}\right)^{\perp}, F\left(\left(U_{n}\right)_{+}, M G L\right)\right] .
$$

Now capping with this element gives the equivalence (7.17).

THEOREM 7.19. If there exists a non-associative division algebra with norm $\left\langle\left\langle a_{1}, \ldots, a_{n-1}\right\rangle\right\rangle$, then $\left\langle\left\langle a_{1}, \ldots, a_{n}\right\rangle\right\rangle$ has the Hopf invariant 1 property. Consequently, every Pfister form with $n \leqslant 4$ has the Hopf invariant 1 property. Further, if $k \subseteq \mathbb{R}$, no unisotropic Pfister form with $n>4$ has the Hopf invariant 1 property.

Proof. By Theorem 7.8 above, if there is a non-associative division algebra with norm $\left\langle\left\langle a_{1}, \ldots, a_{n-1}\right\rangle\right\rangle$, then

$$
U \times(Y-X) \cong U \times\left(Y^{0}-X^{0}\right) .
$$

Hence, $U_{+} \wedge(Y-X)_{+} \cong U_{+} \wedge\left(Y^{0}-X^{0}\right)_{+}$. By definition, in the stable category, the left-hand side is

$$
U_{+} \vee\left(\Sigma^{2^{n-1}(1+\alpha)-1}\left(S^{0}\right)^{\perp} \wedge U_{+}\right)
$$


while the right-hand side is $U_{+} \vee \Sigma^{2^{n-2}(1+\alpha)-1} U_{+}$. This proves the first two statements. To see the last statement, it again suffices to assume that all the coefficients of the Pfister form are +1 . Then consider the Real realization of the spaces involved. Then the realization of $U_{+}$is $S^{2^{n-1} \alpha}$ and, as we saw in Section 4, the realization of $\left(S^{0}\right)^{\perp}$ is $S^{2^{n-1}(\alpha-1)}$. Thus, the Hopf invariant 1 property in this case implies

$$
\Sigma^{2^{n-1}(\alpha-1)} S^{2^{n-1} \alpha} \simeq S^{2^{n-1} \alpha}
$$

in the category of $\mathbb{Z} / 2$-equivariant spaces. Factoring through the action of $\mathbb{Z} / 2$, (7.21) implies an equivalences of stunted projective spaces which only occurs for $n-1=1,2,3$ (see e.g. [8]).

PROBLEM. Which Pfister quadrics have the Hopf invariant 1 property for fields $k$ not contained in $\mathbb{R}$ ?

\section{References}

1. Adams, J. F.: Stable Homotopy and Generalised Cohomology, University of Chicago Press, Chicago, 1974.

2. Araki, S.: Forgetful spectral sequences, Osaka J. Math. 16(1) (1979), 173-199.

3. Araki, S.: Orientations in $\tau$-cohomology theories, Japan. J. Math. (N.S.) 5(2) (1979), 403-430.

4. Artin, M. and Mazur, B.: Etale homotopy, Lecture Notes in Math. 100, Springer-Verlag, New York, 1969.

5. Atiyah, M.: K-theory and reality, Quart. J. Math. Oxford Ser. (2) 17 (1966), 367-386.

6. Brown, E. H. and Peterson, E. P.: A spectrum whose $\mathbb{Z} / p$-cohomology is the algebra of reduced $\mathbb{Z} / p$ powers, Topology 5 (1966), 149-154.

7. Cole, M., Greenlees, J. P. C. and Kriz, I.: The universality of equivariant complex bordism, to appear in Math. Zeit.

8. Davis, D. M. and Mahowald, M.: Classification of the stable homotopy types of stunted real projective spaces, Pacific J. Math. 125(2) (1986), 335-345.

9. Hopkins, M. J. and Morel, F.: Lecture in Tolouse, France, July 2000.

10. Hu, P.: Cobordism of Real manifolds, Proceedings of the 1995 Kazimierz conference in algebraic topology, Fundam. Math. 1999.

11. Hu, P.: $S$-modules in the category of schemes, to appear in Mem. AMS.

12. Hu, P.: On the Picard group of the $\mathbb{A}^{1}$-stable homotopy category, Preprint, 1999.

13. Hu, P. and Kriz, I.: A Real-oriented analogue of the Adams-Novikov spectral sequence, Topology. 40(2) (2001), 317-399.

14. Hurwitz, A.: Über die Komposition der quadratischen Formen, Math. Ann. 88 (1923), 1-25.

15. Jardine, J. F.: $\mathbb{A}^{1}$-local symmetric spectra, Preprint, 1998.

16. Landweber, P. S.: Fixed point free conjugations on complex manif olds, Ann. Math. 86(2) (1967), 491-502.

17. Landweber, P. S.: Conjugations on complex manifolds and equivari ant homotopy of $M U$, Bull. Amer. Math. Soc. 74 (1968), 271-274.

18. Lewis, L. G. Jr., May, J. P. and Steinberger, M.: Equivariant Stable Homotopy Theory. With Contributions by J. E. McClure, Lecture Notes in Math. 1213, Springer-Verlag, New York, 1986.

19. Milne, J. S.: Étale Cohomology, Princeton University Press, 1980. 
20. Mitchell, S.: On the Lichtenbaum-Quillen conjectures from a stable homotopy-thoretic viewpoint, In: Algebraic Topology and its Applications, Math. Sci. Res. Inst. Publ. 27, SpringerVerlag, New York, 1994.

21. Morel, F.: Oral communication, MSRI, May 1998.

22. Morel, F. and Voevodsky, V.: $\mathbb{A}^{1}$-homotopy theory of schemes, Preprint, 1998.

23. Quillen, D.: Higher algebraic $K$-theory I, In: Algebraic K-theory, I: Higher $K$-theories (Proc. Conf., Battelle Memorial Inst., Seattle, Wash., 1972), Lecture Notes in Math. 341, SpringerVerlag, New York, 1972, pp. 85-147.

24. Ravenel, D. C.: Complex cobordism and stable homotopy groups of spheres, Pure Appl. Math. 121, 1986, Academic Press, New York.

25. Rost, M.: The motive of a Pfister form, Preprint, 1998.

26. Rost, M.: On the dimension of a composition algebra. Documenta Math. 1 (1996), 209-214.

27. Voevodsky, V.: Bloch-Kato conjecture for $\mathbb{Z} / 2$-coefficients and algebraic Morava $K$-theories, Preprint 1995

28. Voevodsky, V.: The Milnor conjecture, Preprint, 1996. 